\title{
A New MRP Optimization Algorithm Based on Multi- Objective Genetic Evolution
}

\author{
Qin Liao Wangyu Chen \\ School of Mathematical Science, South China University of Technology, Guangzhou 510640, P. R. China
}

\begin{abstract}
This paper takes integrated optimization of Material Requirements Planning (MRP) and Capacity Requirements Planning (CRP) as research intention, builds a novel multi-objective optimization algorithm of MRP and CRP based on genetic evolution, and designs the proper genetic operators which take the integrated configuration of productive requirement and resource requirement into account. Thereby, it is proved that the novel MRP algorithm based on genetic evolution is superior to the conventional MRP algorithm.
\end{abstract}

Keywords: Genetic algorithm, Multi-objective MRP, CRP

\section{Introduction}

Material Requirements Planning (MRP) of conventional Enterprise Resource Planning (ERP) is based on "stock cost" and assigns productive tasks according to the goal that neither out of stock (OOS) nor overstock would happen. Then Capacity Requirements Planning (CRP) assigns the required force and machine resource for productive tasks. Because the conventional MRP algorithm does not consider productive resource directly, it would usually result in the situation that MRP plan has to be rebuilt repeatedly on account of the exceeded productive capacity in CRP, which could not support the MRP plan. This repeated modifications lead to low usage of CRP with resource configuration in practice. However, the research about this problem is still insufficient at present.

This paper takes the integration and consistency of productive resource as a goal, integrates genetic algorithm with the MRP and CRP respectively in single production and multi-production situation. On one hand this method holds the original advantage of "neither out of stock nor overstock", On the other hand it optimizes the configuration of productive requirement, productive plan and productive resource. The optimization process not only takes the stock cost as standard evaluation but also takes OOS cost and productive cost as integrative evaluation, which makes the optimized plan of MRP and CRP more useful.

\section{Improvement and optimization of single MRP algorithm}

\subsection{Problems of MRP algorithm}

Assume the variables as follows:

POH: projected on-hand, OH: on hand, AL: allocation, NR: net requirement, PORC: planned order receipt, PAB: projected available balance, SR(0): SR exceeds the time limit, SR: scheduled receipts, n: the number of periods, POR: planned order release. LT: lead time, UMH: man hour of unit production, PTH: the total man hour of plan, FTH: the actual total man hour, LR: load ratio.

Conventional MRP algorithm process [4]:

step $1: P O H(t)=\left\{\begin{array}{lc}O H+S R(t)+\max \{S R(0), 0\}-A L-G R(t) & t=1 \\ P A B(t-1)+S R(t)-G R(t) & 1<t \leq n\end{array}\right.$

step $2: N R(t)=\left\{\begin{array}{ll}0 & , P O H(t) \geq S S \\ S S-P O H(t) & , P O H(t)<S S\end{array} \quad 1 \leq t \leq n\right.$

step $3: P O R C(t)=\left\{\begin{array}{ll}\max \{N R(t), L S\} & , N R(t)>0 \\ 0 & , N R(t) \leq 0\end{array} \quad 1 \leq t \leq n\right.$

step 4: $P A B(t)=P O H(t)+P O R C(t) \quad 1 \leq t \leq n$

step 5:POR $(t)=$ PORC $(t+L T) \quad 1 \leq t \leq n$

Conventional CRP algorithm process [4]:

$P T H(t)=U M H \times P O R(t) \quad 1 \leq t \leq n$

$L R(t)=\frac{P T H(t)}{F T H} \times 100 \% \quad 1 \leq t \leq n$

The productive capacity is exceeded when LR $>100 \%$, in that situation more resources are needed to solve the productive problem of plan.

To ensure the consistence of MRP and CRP, both restriction and configuration of resource must directly be performed in the MRP algorithm. Suppose on the basis of the conventional MRP algorithm, genetic algorithm with factors of MRP and CRP is introduced to find the best production plan, which minimizes the cost and makes the best use of productive resource in the fixed productive requirement situation. The optimized process in this paper is that PORC is searched by genetic algorithm instead of generated by step3. 


\subsection{Integration algorithm of MRP and CRP based on genetic evolution}

\subsubsection{Genetic encoding designment of $M R P$ and $C R P$}

Assumed that $O H, A L, R G, S R, N R$ are given, $P O R$ can be expressed by $P O R C$ while $P O H$ and $P O R C$ can be expressed by each other. So PORC of MRP can be directly taken as the individual of genetic algorithm. It means that the $i$ th period of PORC is marked as gene $x_{i}$, and the individual of PORC with $n$ periods are marked as $X=\left(x_{1}, x_{2}, \ldots, x_{n}\right), x_{i} \in \square$

\subsubsection{Genetic population of MRP and CRP}

Based on the original individual $X\left(x_{1}, x_{2}, \ldots, x_{n}\right)$ generated by conventional MRP algorithm, choose an element $c$ randomly from $\{c: L S \times n,|n| \leq \varepsilon, n \in \square\}$ for each $x_{i}$, then let $x_{1 i}=x_{i}+c$. The new $x_{1 i}, i=1, \ldots, n$ make up a new individual $X_{1}\left(x_{11}, x_{12}, \ldots, x_{1 n}\right)$, follow the same steps to generate $m-1$ individuals, and at last an original population $\mathrm{Z}=\left\{X, X_{1}, \ldots, X_{m-1}\right\}$ is created. Because the original individual generated by conventional MRP algorithm is one member of population, the optimized individual $(P O R C)$ must be better than the original individual (PORC). Where, $L S$ : Lot Sizing, $\varepsilon$ : adjusted range, $m$ : population size. Each gene $x_{i}$ must be the multiple of $L S$, which ensures the feasibility of algorithm result. It is desired that $\varepsilon \in \square^{+}$and it must be enough to correct the maximal overload. And when the original population is created, there are 3 conditions to be satisfied:

1. $x_{i t} \geq 0, i=1,2, \ldots, m ; t=1,2, \ldots, n$

2. $P A B_{i t} \geq S S, i=1,2, \ldots, m ; t=1,2, \ldots, n$

3. $\sum_{i=1}^{n} x_{i t} \leq n \times \frac{F T H}{U M H}, i=1,2, \cdots, m$;

The individual of population that satisfies the 3 conditions above would be more feasible. So the searching is limited in the feasible region.

\subsubsection{Fitness function definition of MRP and $C R P$}

The fitness function is defined on the basis of the cost control and capacity overload.

1. Capacity overload control function $L(x)$

$$
\begin{aligned}
& L(X)=\sum_{t=1}^{n} l\left(x_{t}\right) \\
& l\left(x_{t}\right)=\frac{x_{t} \times U M H}{F T H}, \quad \frac{x_{t} \times U M H}{F T H}>1,1 \leq t \leq n
\end{aligned}
$$

Where $X$ is an individual. The more periods of overload or the more productions of overload are, the bigger $L(X)$ would be, vice versa.

2. Normal load control function $F U L L(X)$

$$
\operatorname{FULL}(X)=\sum_{t=1}^{n} \operatorname{full}\left(x_{t}\right)
$$

full $\left(x_{t}\right)=\left\{\begin{array}{lll}\frac{F T H}{U M H}-x_{t} & , 0<x_{t} \leq \frac{F T H}{U M H} & \\ 0 & , x_{t}=0 & 1 \leq t \leq n \\ 5 \times \frac{F T H}{U M H} & , x_{t}>\frac{F T H}{U M H} & \end{array}\right.$

Where $F U L L(X)$ denotes the level of normal load. If production is less than maximal production, $F U L L(X)$ would be bigger, vice versa. It is extra assumed that $F U L L(X)=5 \times \frac{F T H}{U M H}$ when $x_{t}>\frac{F T H}{U M H}$ for much stronger control of overload because the numerical value of $L(x)$ above is far less than $F U L L(X)$. 3. Cost control function

- $\quad$ Stock cost control function $H(X)$

$H(X)=\sum_{t=1}^{n} h\left(x_{t}\right)$

$h\left(x_{t}\right)=V S C \times P A B_{t}+F S C, \quad 1 \leq t \leq n$

Where $V S C$ is variable stock unit cost, FSC is fixed stock unit cost. $H(X)$ would be bigger if $P A B$ is bigger, vice versa.

- . Productive cost control function $P(X)$

$P(X)=\sum_{t=1}^{n} p\left(x_{t}\right)$

$p\left(x_{t}\right)=V P C \times x_{t}+F P C, \quad 1 \leq t \leq n$

Where $X$ is an individual, $V P C$ is variable productive unit cost; FSC is fixed productive unit cost. $P(X)$ would be bigger if $\operatorname{PROC}\left(x_{t}\right)$ is bigger, vice versa.

4. Genetic fitness function $\operatorname{Fitness}(X)$

$$
\begin{aligned}
& f i t(X)=\alpha \times L(X)+\beta \times F U L L(X)+\gamma \times H(X)+\delta \times P(X) \\
& \operatorname{Fitness}(X)=\frac{1}{f i t(X)+\sigma}
\end{aligned}
$$

Where $\alpha, \beta, \gamma, \delta$ are weights and $\sigma$ is an arbitrary small positive number. If the adaptability of an individual is higher, Fitness $(X)$ would be bigger, vice versa.

\subsubsection{Genetic algorithm process of MRP and CRP}

This paper takes elitist selection and expectation selection as selection operators, takes uniformity crossover as the crossover operator and uniformity mutation as the mutation operator. The optimized integrated genetic algorithm process is:

1. Use steps of 2.2 .2 to generate $m$ individuals which satisfy $(8)(9)(10)$ to buildup the initial population $Z$.

2. Calculate Fitness $\left(X_{k}\right)$ for each $X_{k}$ based on (16).

3. Select the good individuals according to Fitness $\left(X_{k}\right)$, and implement uniformity crossover and mutation.

If the result does not achieve the expectation, go to step 2; if it does, terminate the algorithm process, the best individual is the one whose fitness value is biggest and corresponding PROC makes cost minimal and productive resource best used. 


\subsection{Comparison between the optimized result and original result of MRP and CRP}

\subsubsection{Evaluation definition}

According to the optimized goal and features of genetic algorithm, this paper evaluates the result from several aspects below in order to show the difference between original plan and optimized plan.

1. The improved ratio of fitness $(I R F)$

$I R F=\frac{B F-O F}{O F} \times 100 \%$

Where $B F$ is the fitness of best individual, $O F$ is the fitness of the individual corresponding original MRP plan.

2. The improved ratio of overload (IROL)

IROL $=\frac{O L(X)-B L(X)}{O L(X)} \times 100 \%$

Where $O L(X)$ is the value of capacity overload control function $L(X)$ about original plan and $B L(X)$ is the one of optimized plan.

3. The improved ratio of normal load (IRNL)

$f p\left(x_{t}\right)=\left\{\begin{array}{lc}\frac{x_{t} \times U M H}{F T H}, & 0.5<\frac{x_{t} \times U M H}{F T H} \leq 1 \\ 0, & \frac{x_{t} \times U M H}{F T H} \leq 0.5\end{array}\right.$

$R N L(X)=\sum_{i=1}^{n} f p\left(x_{i}\right)$

$I R N L=\frac{B R N L-O R N L}{O R N L} \times 100 \%$

Where $R N L$ is the ratio of total normal load, $B R N L$ is the $R N L$ of optimized plan; ORNL is the $R N L$ of original plan.

4. The improved ration of total cost (IRTC)

$I R S C=\frac{O S C-B S C}{O S C} \times 100 \%$

$I R P C=\frac{O P C-B P C}{O P C} \times 100 \%$
$I R T C=\frac{(O S C+O P C)-(B S C+B P C)}{O S C+O P C} \times 100 \%$

Where IRSC is the improved ratio of stock cost, $I R P C$ is the improved ratio of productive cost; OSC is stock cost of original plan while $B S C$ is stock cost of optimized plan; $O P C$ is productive cost of original plan while $B P C$ is productive cost of optimized plan.

\subsubsection{Optimized example}

Here, MRP plan of Table4 is taken as an optimized example. The requirement in MRP plan of materiall is stable and regular. Capacity and load is out of balance in the 3rd, 5th, 7th, 9th, 11th period of MRP plan when $F T H_{1}=75, U M H=0.5$, the $P R O C$ exceeds the maximal capacity in that period while normal load ratio of PROC in other periods do not achieve $100 \%$.

1. Parameter setting

Max generation: 200; Population size: 50;

Adjusted range $\varepsilon: 3$;

Crossover probability $p_{c}: 0.85$

Mutation probability $p_{m}: 0.009$;

Selection operator: expectation method;

Crossover operator: uniformity crossover;

Mutation operator: uniformity mutation;

Weight of fitness function: $\alpha=0.5, \beta=0.3, \gamma=0.1, \delta=0.1$;

2. The optimized result

There are 5 overload periods in original plan of Table 1, both MRP and CRP plans need to be modified after the assignment of CRP. After optimization, production in 5 overload periods is balanced; what's more, there are 9 periods whose normal load ratio achieves $100 \%$ and MRP and CRP's plans do not need to be modified. "Original" in Table 2 denotes original MRP plan; "Optimized" in Table 2 denotes optimized MRP plan. It can be seen from Table 2 that: IROL declines by $100 \%$; IRNL is increased by $210 \%$; IRTC declines by $0.54 \%$.

\begin{tabular}{|c|c|c|c|c|c|c|c|c|c|c|c|c|c|}
\hline Period & 0 & 1 & 2 & 3 & 4 & 5 & 6 & 7 & 8 & 9 & 10 & 11 & 12 \\
\hline \multicolumn{14}{|l|}{ Orginal } \\
\hline GR & & 100 & 200 & 100 & 200 & 100 & 200 & 100 & 200 & 100 & 200 & 100 & 200 \\
\hline PORC & & 0 & 50 & 100 & 200 & 100 & 200 & 100 & 200 & 100 & 200 & 100 & 200 \\
\hline$\overline{\mathrm{PAB}}$ & & 260 & 110 & 110 & 110 & 110 & 110 & 110 & 110 & 110 & 110 & 110 & 110 \\
\hline PTH & 0 & 25 & 50 & 100 & 50 & 100 & 50 & 100 & 50 & 100 & 50 & 100 & \\
\hline LR (\%) & 0 & 33.3 & 66.6 & 133 & 66.6 & 133 & 66.6 & 133 & 66.6 & 133 & 66.6 & 133 & \\
\hline \multicolumn{14}{|l|}{ Result } \\
\hline PORC & & 0 & 150 & 100 & 150 & 100 & 150 & 150 & 150 & 150 & 150 & 150 & 150 \\
\hline PAB & & 260 & 210 & 210 & 160 & 160 & 110 & 160 & 110 & 160 & 110 & 160 & 110 \\
\hline PTH & 0 & 75 & 50 & 75 & 50 & 75 & 75 & 75 & 75 & 75 & 75 & 75 & \\
\hline LR (\%) & 0 & 100 & 66.6 & 100 & 66.6 & 100 & 100 & 100 & 100 & 100 & 100 & 100 & \\
\hline
\end{tabular}

Table 1: Result of single material 1' MRP and CRP. 


\begin{tabular}{|l|l|l|l|l|l|l|l|}
\hline & Original & Optimized & Improved & & Original & Optimized & Improved \\
\hline Fitness & 0.0000559 & 0.0000597 & $6.64 \%$ & Stock Cost & 14940 & 15840 & $-6.02 \%$ \\
\hline Overload & 0.5555 & 0 & $100 \%$ & Product Cost & 151000 & 151000 & $0 \%$ \\
\hline Normal Load & 3.3333 & 10.3333 & $210 \%$ & Total Cost & 165940 & 166840 & $-0.54 \%$ \\
\hline
\end{tabular}

Table 2: Result of the optimization.

\section{Improvement and optimization of multi-MRP algorithm}

Conventional multi-MRP algorithm generates the MRP plans for each production and accumulates the required capacity for each period of all productions, then creates the CRP plan consequently. The error of the accumulative capacity is quite large when many productions' MRP plans exceed capacity in the same period, and that is why the usage of CRP is low. To integrate and optimize multi-MRP and CRP algorithm, a multi-objective programming model for MRP with $n$ kinds of productions is built. It differs from single MRP algorithm that it needs to consider OOS cost besides stock cost and productive cost for the optimization result evaluation. When information is not adequate, weights of goals are hard to be set up. Lexicographic ordering approach [3], which is based on the hierarchy relation of goals, and adaptive weight approach [3], which can automatically adjust weights according to the relation between goals of individual and limit point, are utilized to design fitness function to avoid the problem above. $(\mu+\lambda)$ selection [2] is used to design selection operator and $N$ points' crossover and mutation operators are designed according to $N$ kinds of production. All above make the searching of optimized solution more effectively.

\subsection{Designment of multi-MRP and CRP algorithm}

\subsubsection{Multi-objective programming model}

Suppose that there are $N$ kinds of productions and $T_{i}$ periods in $i$ th production. The integrated and optimized process of MRP and CRP in multiproduction is to minimize the productive cost, stock cost and OOS cost, make the best use of productive resource and implement productive plan. The multiobjective programming model of multi-MRP and CRP is:

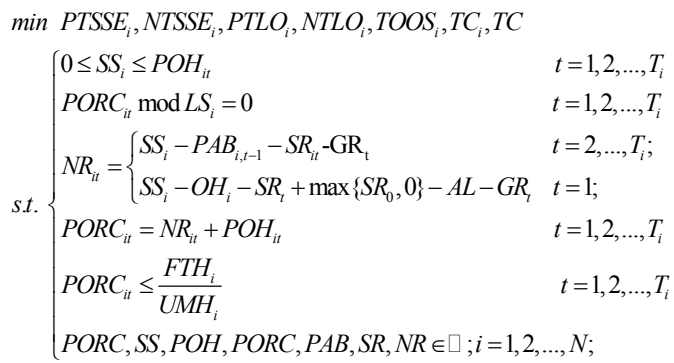

1. The $1 \mathrm{st}$ restriction means that $P O H$ of $i$ th production and $t$ th period can not be lower than safety stock, $S S_{i}$ is safety stock of $i$ th production, and the total safety stock error (TSSE) of $i$ th production is defined according to this restriction:

The positive total safety stock error of $i$ th production:

$\operatorname{PTSSE}_{i}=\sum_{t=1}^{T} S S E_{i t}^{+}, \quad$ while $S S E_{i t}^{+}=P O H_{i t}-S S_{i}\left(P^{\prime} O H_{i t}-S S_{i} \geq 0\right)$

The negative total safety stock error of $i$ th production:

$N T S S E_{i}=\sum_{i=1}^{T} S S E_{i t}^{-}, \quad$ while $S S E_{i t}^{-}=S S_{i}-P O H_{i t}\left(P^{\prime} O H_{i t}-S S_{i}<0\right)$

2. The 2nd restriction means that PORC of $i$ th production and $t$ th period must be multiple of $L S$ of $i$ th production.

3. The 3rd restriction means the relation of NR, SS, $\mathrm{POH}, \mathrm{PORC}, \mathrm{AL}$ and GR of $i$ th production and $t$ th period.

4. The 4th restriction means the relation of $P O R C$, $N R$ and $P O H$ of $i$ th production and $t$ th period, The total out of stock(TOOS) of $i$ th production is defined according to this restriction:

$\operatorname{TOOS}_{i}=\sum_{t=1}^{T} \operatorname{OOS}_{i t}$, while $\operatorname{OOS}_{i t}=-P O H_{i t}\left(P O H_{i t}<0\right)$

5. The 5th restriction means the production of $i$ th production and $t$ th period must be less than its max capacity. And the total load error $(T L O)$ is defined according to this restriction:

The positive total load error of $i$ th production:

PTLO $_{i}=\sum_{k=1}^{T} L O_{i k}^{+}$, while $L O_{i t}^{+}=$PORC $_{i t}-\frac{F T H_{i}}{U M H_{i}}\left(\right.$ PORC $\left._{i t}-\frac{F T H_{i}}{U M H_{i}} \geq 0\right)$

The negative total load error of $i$ th production:

$N T L O_{i}=\sum_{k=1}^{T} L O_{i k}^{-}, \quad$ while $L O_{i t}^{-}=\frac{F T H_{i}}{U M H_{i}}-P O R C_{i t}\left(P^{\prime} O R C_{i t}-\frac{F T H_{i}}{U M H_{i}}<0\right)$

6. The total cost of $i$ th production $\left(T C_{i}\right)$ :

$T C_{i}=F C_{i}+P C_{i}+S C_{i}+O O S C_{i}$

Where $P C_{i}$ is the productive cost of $i$ th production and $C_{i l}$ is the unit productive cost of $i$ th production:

$P C_{i}=C_{i 1} \sum_{t=1}^{T} P O R C_{i t}$ 
$S C_{i}$ is the stock cost of $i$ th production and $C_{i 2}$ is the unit stock cost of $i$ th production:

$$
S C_{i}=C_{i 2} \sum_{t=1}^{T} S_{i t}^{+} \text {, while } S_{i t}^{+}=P O H_{i t}\left(P O H_{i t}>0\right)
$$

$\mathrm{OOSC}_{i}$ is OOS cost of $i$ th production and $C_{i 3}$ is the unit OOS cost of $i$ th production:

$$
\mathrm{OOSC}_{i}=C_{i 3} \sum_{t=1}^{T} \operatorname{OOS}_{i t} \text { while OOS } i t=-\mathrm{POH}_{i t}\left(\mathrm{POH}_{i t}<0\right)
$$

7. Total cost of $N$ productions (TC):

$$
T C=\sum_{i=1}^{N} T C_{i}
$$

\subsubsection{Solving multi-objective programming model of ERP and CRP with genetic algorithm}

\section{Genetic population of multi-MRP and CRP}

Assume $x_{i j}$ denotes the PORC of $i$ th production and $j$ th period and then PORC of $i$ th production and $T_{i}$ period should be $A_{i}=\left(x_{i 1}, x_{i 2}, \cdots x_{i T_{i}}\right)$, the individual made up of $N$ kinds of productions should be:

$X=\left(A_{1}, A_{2}, \cdots A_{N}\right)=\left(x_{11}, \cdots x_{1 T_{1}} ; x_{21}, \cdots x_{2 T_{2}} ; x_{N 1} \cdots x_{N T_{N}}\right)$

Obtain $x_{i j}$ from $\left\{c: L S_{i} \times n,\left|n-\frac{F T H_{i}}{U M H_{i}}\right| \leq \varepsilon, n \in \square\right\}$ randomly to

build up individual $X^{(K)} \quad k=1,2, \ldots, M ; M$ individuals build up population $\left\{X^{(1)}, X^{(2)}, \cdots, X^{(M)}\right\}$;

2. Fitness function designment of multi-MRP and CRP

This paper utilizes lexicographic ordering approach [3] and adaptive weight approach [3] to define the genetic fitness function respectively.

- Lexicographic ordering approach (LOA)

LOA is to make individuals sorted by the lexicographic ordering according to the goals. That is to sort the population by the 1st priority goal, when there is the same value of the 1st goal, sort them by the 2nd priority goal; and at last if there is the same value of all goals, they should be sorted randomly. After the sorting work from best to worst above, the fitness is assigned to all individuals.

For goal sequence of model (24): PTSSE $_{i}, N_{T S S E}$, $P T L O_{i}, N_{T L O}, \operatorname{TOOS}_{i}, T_{i}, T C(i=1, \cdots, n)$, the key to this problem is how to set up the priority for the 7 kinds of $6 N+1$ goals. There are 3 kinds of sorting methods according to the production importance and the kinds of goals.

(1) Sort goals by 7 kinds firstly, and then sort goals of each kind by the production importance.

(2) Sort goals by the production importance firstly, then sort goals of each production by the kinds of goals.

(3) Based on the method (2), get the 1 st priority goal of each production together and sort them by the production importance, then get the 2 nd priority goal of each production together and sort them by by the production importance also until the 7 th priority goal, then put these goals together in turn.

- $\quad$ Adaptive weight approach (AWA)

AWA is an approach that adjusts the weights of multi-goals automatically according to the relation between goals of individual and maximal and minimal limit point in evolving process. According to the character of this problem, this paper defines the minimal limit point of all the generations until now instead of the one of current generation as the minimal limit point of the algorithm. This change makes the searching of optimized global solution more effectively.

Assume the goals' sequence of model (24) in turn as:

$$
Z_{1}=f_{1}(A), Z_{2}=f_{2}(A), \ldots, Z_{6 N+1}=f_{6 N+1}(A)
$$

Suppose $P^{(k)}$ is the $k$ th generation and define maximal limit point $Z^{+}$and minimal limit point $Z$ as follows:

$$
\begin{gathered}
Z^{+}=\left(Z_{1}^{\max }, Z_{2}^{\max }, \ldots, Z_{6 N+1}^{\max }\right), Z_{i}^{\max }=\max \left\{f_{i}(A) \mid A \in P^{(k)}\right\}, \\
i=1,2, \ldots, 6 N+1 \\
Z^{-}=\left(Z_{1}^{\min }, Z_{2}^{\min }, \ldots, Z_{6 N+1}^{\min }\right), Z_{i}^{\min }=\min \left\{f_{i}(A) \mid A \in \bigcup_{j=1}^{k} P^{(j)}\right\}, \\
i=1,2, \ldots, 6 N+1
\end{gathered}
$$

Then the object function is:

$Z(A)=\sum_{i=1}^{6 N+1} \frac{Z_{i}^{\max }-Z_{i}}{Z_{i}^{\max }-Z_{i}^{\min }}$

The punish function is:

$P(A)=1-\frac{1}{6 N-1} \sum_{i=1}^{6 N+1}\left(\frac{Z_{i}}{Z_{i}^{\max }}\right)$

So the fitness function is:

$\operatorname{eval}(A)=Z(A) P(A)$

3. Genetic operator designment of multi-MRP and CRP

- . $(\mu+\lambda)$ selection

The main idea of $(\mu+\lambda)$ selection is to let the offspring individuals compete with previous ones in order to avoid that offspring individuals substitute the previous directly. The detailed process is that let the offspring individuals and previous individuals compete with each other and sorted from best to worst. (Suppose the previous population size is $\mu$ and the offspring population size is $\lambda$ ), then take the best $\mu$ individuals as the new population.

There are 3 advantages of $(\mu+\lambda)$ selection:

(1) Some best individuals will not miss because the offspring individuals do not substitute the previous ones.

(2) Because previous individuals and offspring ones are both reserved, the selection space is enlarged.

(3) The new population will be made more sense because previous individuals and offspring ones compete with each other.

- $\quad \mathrm{N}$ points' crossover and mutation 
$N$ points' crossover is to select a gene location for each $A_{i}$ of two individuals and exchange all the genes from the gene location in $A_{i}$ with crossover probability $p_{c}$, which create two new individuals. The 2 new individuals will be reserved if they both satisfy $\operatorname{NTSSE}_{i}=0$ and $\operatorname{TOOS}_{i}=0$ for $i=1,2, \cdots, n$; otherwise, they would be abandoned directly.

$N$ points' mutation is to choose $c_{i k}$ from $\left\{c: L S_{i} \times n,|n| \leq \varepsilon, n \in \square\right\} \quad$ randomly for each $a_{i k}$ in $\left(a_{11}, \cdots a_{1 T_{1}} ; a_{21}, \cdots a_{2 T_{2}} ; a_{N 1} \cdots a_{N T_{N}}\right)$ and let $b_{i k}=a_{i k}+c_{i k}$. Then a new individual $\left(b_{11}, \cdots b_{1 T_{1}} ; b_{21}, \cdots b_{2 T_{2}} ; b_{N 1} \cdots b_{N T_{N}}\right)$ is created, where the setting of $L S_{i}$ and $\varepsilon$ is the same with 2.2.2. if the new individual satisfies 3 restriction below, it will be reserved; otherwise, it would be abandoned directly.

(1) $b_{i t} \geq S S_{i}$

(2) $\sum_{t=1}^{T_{i}} \mathrm{PORC}_{i t} \leq T_{i} \times \frac{\mathrm{FTH}_{i}}{\mathrm{UMH}_{i}} \quad i=1,2, \ldots, N$

(3) $\operatorname{NTSSE}_{i}=0$ and $\operatorname{TOOS}_{i}=0$ for $i=1,2, \cdots, n$;

\subsection{Comparison between the optimized result and original result of multi-MRP and CRP}

The multi-MRP and CRP algorithm process is the same with 2.2.4, and the only difference is that the definition of individual, fitness function, crossover and mutation operator are based on 3.1.2.

\subsubsection{Evaluation definition}

There are more aspects to be added to evaluate the optimized result besides aspects in 2.3.1 for the multiMRP and CRP algorithm.

1. The improved ratio of positive safety stock error of $i$ th production $\left(I_{R P S S E}\right)$

$I_{R P S S E}=\frac{O P T S S E_{i}-B P T S S E_{i}}{B P T S S E_{i}} \times 100 \%$

Where $O P T S S E_{i}$ denotes the PTSSE $E_{i}$ of original plan of $i$ th production, BPTSSE $_{i}$ denotes the $P T S S E_{i}$ of optimized plan.

2. The improved ratio of negative safety stock error of $i$ th production $\left(I R N S S E_{i}\right)$

$I_{R N S S E}=\frac{\text { ONTSSE }_{i}-\text { BNTSSE }_{i}}{B N T S S E_{i}} \times 100 \%$
Where $O N T S S E_{i}$ denotes the NTSSE $E_{i}$ of original plan of $i$ th production, $B N T S S E_{i}$ denotes the $N T S S E_{i}$ of optimized plan.

3. The improved ratio of overload cost of $i$ th production $\left(I R O L C_{i}\right)$

$$
I_{R O L C}=\frac{O T O L C_{i}-\text { BTOLC }_{i}}{\text { OTOLC }_{i}} \times 100 \% \text {, while TOLC } C_{i}=5 \times C_{i 1} \times T_{L} O_{i}^{+}
$$

Where $T O L C_{i}$ is the overload cost of $i$ th production, $O T O L C_{i}$ is $T O L C_{i}$ of original plan and $B T O L C_{i}$ is $T O L C_{i}$ of optimized plan.

4. The improved ratio of total cost of $N$ productions (IRTCN)

$$
\operatorname{IRTCN}=\frac{\sum_{i=1}^{N}\left(O P C_{i}+O S C_{i}+O T O L C_{i}\right)-\sum_{i=1}^{N}\left(B P C_{i}+B S C_{i}+B T O L C_{i}\right)}{\sum_{i=1}^{N}\left(O P C_{i}+O S C_{i}+O T O L C_{i}\right)} \times 100 \%
$$

Where $P C_{i}, S C_{i}$ and $T O L C_{i}$ have been defined before.

\subsubsection{Optimized examples}

Material 1 in table 3 has been introduced in 2.3.2.

The requirement of Material 2 in table 4 is fluctuant periodically. Capacity and load are out of balance in the $2 \mathrm{nd}, 4 \mathrm{th}$, 9 th period of MRP plan when $F_{T H}=100, \quad U M H=0.2$, the PROC exceeds the maximal capacity in that period while normal load ratio of PROC in other periods do not achieve $100 \%$.

\section{Parameter setting}

Max generation: 200; Population size: 50;

Adjusted range $\varepsilon: 3$;

Crossover probability $p_{c}: 0.85$

Mutation probability $p_{m}: 0.009$;

2. The optimized result

After optimization, production of 5 overload periods in original plan of Table 5 are balanced; what's more, there are 11 periods whose normal load ratio achieves $100 \%$ and MRP and CRP's plans do not need to be modified.

After optimization, production of 3 overload periods in original plan of Table 6 are balanced; what's more, there are 5 periods whose normal load ratio achieves $100 \%$ and MRP and CRP's plans do not need to be modified.

IRTCN of these two materials declines by about $24 \%$, and there are different improvements of productive cost, stock cost and load (Table 8), both IROL of them declines by $100 \%, I R N L$ of material 1 is increased by $136 \%$ and $I R N L$ of material 2 is increased by $120 \%$.

\begin{tabular}{|l|l|r|r|r|r|r|r|r|r|r|r|r|r|r|}
\hline & & \multicolumn{2}{|c|}{$\mathrm{OH}_{1}=180$} & \multicolumn{2}{|c|}{$\mathrm{LT}_{1}=1$} & \multicolumn{2}{c|}{$\mathrm{LS}_{1}=50$} & \multicolumn{2}{c|}{$\mathrm{AL}_{1}=0$} & \multicolumn{2}{|c|}{$\mathrm{SS}_{1}=100$} & \multicolumn{2}{c|}{$\mathrm{FTH}_{1}=75$} \\
\hline Period & 0 & 1 & 2 & 3 & 4 & 5 & 6 & 7 & 8 & 9 & 10 & 11 & 12 \\
\hline GR & -100 & 100 & 200 & 100 & 200 & 100 & 200 & 100 & 200 & 100 & 200 & 100 & 200 \\
\hline SR & -50 & 180 & & & & & & & & & & & \\
\hline POH & & 260 & 60 & 10 & -90 & 10 & -90 & 10 & -90 & 10 & -90 & 10 & -90 \\
\hline PAB & & 260 & 110 & 110 & 110 & 110 & 110 & 110 & 110 & 110 & 110 & 110 & 110 \\
\hline NR & & 0 & 40 & 90 & 190 & 90 & 190 & 90 & 190 & 90 & 190 & 90 & 190 \\
\hline
\end{tabular}




\begin{tabular}{|l|l|l|l|l|l|l|l|l|l|l|l|l|l|}
\hline PORC & & 0 & 50 & 100 & 200 & 100 & 200 & 100 & 200 & 100 & 200 & 100 & 200 \\
\hline POR & & 50 & 100 & 200 & 100 & 200 & 100 & 200 & 100 & 200 & 100 & 200 & \\
\hline PTH & 0 & 25 & 50 & 100 & 50 & 100 & 50 & 100 & 50 & 100 & 50 & 100 & \\
\hline LR (\%) & 0 & 33.3 & 66.6 & 133 & 66.6 & 133 & 66.6 & 133 & 66.6 & 133 & 66.6 & 133 & \\
\hline
\end{tabular}

Table 3: Form of Material 1's MRP and CRP.

\begin{tabular}{|l|l|l|l|l|l|l|l|l|l|l|l|}
\hline $\mathrm{OH}_{2}=0$ & \multicolumn{3}{l}{$\mathrm{LT}_{2}=1$} & \multicolumn{2}{l|}{$\mathrm{LS}_{2}=50$} & $\mathrm{AL}_{2}=150$ & \multicolumn{2}{l|}{$\mathrm{SS}_{2}=0$} & \multicolumn{2}{l|}{$\mathrm{FTH}_{2}=100$} \\
\hline Period & 0 & 1 & 2 & 3 & 4 & 5 & 6 & 7 & 8 & 9 & 10 \\
\hline GR & 0 & 890 & 10 & 590 & 190 & 590 & 190 & 590 & 10 & 590 & 590 \\
\hline SR & 600 & 300 & & & & & & & & & \\
\hline POH & & -140 & -50 & -540 & -130 & -520 & -110 & -500 & -10 & -500 & -590 \\
\hline PAB & & 60 & 50 & 60 & 70 & 80 & 90 & 0 & 90 & 0 & 0 \\
\hline NR & & 140 & 0 & 540 & 130 & 520 & 110 & 500 & 10 & 500 & 590 \\
\hline PORC & & 200 & 0 & 600 & 200 & 600 & 200 & 500 & 100 & 500 & 600 \\
\hline POR & 200 & 0 & 600 & 200 & 600 & 200 & 500 & 100 & 500 & 600 & \\
\hline PTH & 40 & 0 & 120 & 40 & 120 & 40 & 100 & 20 & 100 & 120 & \\
\hline LR (\%) & 40 & 0 & 120 & 40 & 120 & 40 & 100 & 20 & 100 & 120 & \\
\hline
\end{tabular}

Table 4: Form of Material 2‘s MRP and CRP.

\begin{tabular}{|c|c|c|c|c|c|c|c|c|c|c|c|c|c|c|}
\hline & Period & 0 & 1 & 2 & 3 & 4 & 5 & 6 & 7 & 8 & 9 & 10 & 11 & 12 \\
\hline \multirow[t]{4}{*}{ Original } & PAB & & 180 & 110 & 110 & 110 & 110 & 110 & 110 & 110 & 110 & 110 & 110 & 110 \\
\hline & POR & 0 & 50 & 100 & 200 & 100 & 200 & 100 & 200 & 100 & 200 & 100 & 200 & \\
\hline & PTH & 0 & 25 & 50 & 100 & 50 & 100 & 50 & 100 & 50 & 100 & 50 & 100 & \\
\hline & LR (\%) & 0 & 33.3 & 66.6 & 133 & 66.6 & 133 & 66.6 & 133 & 66.6 & 133 & 66.6 & 133 & \\
\hline \multirow[t]{4}{*}{ LOA } & PAB & & 310 & 110 & 160 & 110 & 160 & 110 & 160 & 110 & 160 & 110 & 160 & 110 \\
\hline & POR & 0 & 0 & 150 & 150 & 150 & 150 & 150 & 150 & 150 & 150 & 150 & 150 & \\
\hline & PTH & 0 & 0 & 75 & 75 & 75 & 75 & 75 & 75 & 75 & 75 & 75 & 75 & \\
\hline & LR (\%) & 0 & 0 & 100 & 100 & 100 & 100 & 100 & 100 & 100 & 100 & 100 & 100 & \\
\hline \multirow[t]{4}{*}{ AWA } & PAB & & 160 & 110 & 160 & 110 & 160 & 110 & 160 & 110 & 160 & 110 & 160 & 110 \\
\hline & POR & 0 & 150 & 150 & 150 & 150 & 150 & 150 & 150 & 150 & 150 & 150 & 150 & \\
\hline & PTH & 0 & 75 & 75 & 75 & 75 & 75 & 75 & 75 & 75 & 75 & 75 & 75 & \\
\hline & LR (\%) & 0 & 100 & 100 & 100 & 100 & 100 & 100 & 100 & 100 & 100 & 100 & 100 & \\
\hline
\end{tabular}

Table 5: Comparison between original plan and optimized plan of material 1 in multi-MRP and CRP.

\begin{tabular}{|c|c|c|c|c|c|c|c|c|c|c|c|c|}
\hline & Period & 0 & 1 & 2 & 3 & 4 & 5 & 6 & 7 & 8 & 9 & 10 \\
\hline \multirow[t]{4}{*}{ Original } & PAB & & 60 & 50 & 60 & 70 & 80 & 90 & 0 & 90 & 0 & 0 \\
\hline & POR & 200 & 0 & 600 & 200 & 600 & 200 & 500 & 100 & 500 & 600 & \\
\hline & PTH & 40 & 0 & 120 & 40 & 120 & 40 & 100 & 20 & 100 & 120 & \\
\hline & LR (\%) & 40 & 0 & 120 & 40 & 120 & 40 & 100 & 20 & 100 & 120 & \\
\hline \multirow[t]{4}{*}{ LOA } & PAB & & 110 & 100 & 10 & 120 & 30 & 90 & 0 & 140 & 50 & 10 \\
\hline & POR & 0 & 500 & 300 & 500 & 250 & 500 & 150 & 500 & 550 & 0 & \\
\hline & PTH & 0 & 100 & 60 & 100 & 50 & 100 & 30 & 100 & 110 & 0 & \\
\hline & LR (\%) & 0 & 100 & 60 & 100 & 50 & 100 & 30 & 100 & 110 & 0 & \\
\hline \multirow[t]{4}{*}{ AWA } & PAB & & 10 & 100 & 10 & 120 & 30 & 90 & 0 & 190 & 100 & 10 \\
\hline & PORC & 100 & 500 & 300 & 500 & 250 & 500 & 200 & 500 & 500 & 0 & \\
\hline & PTH & 20 & 100 & 60 & 100 & 50 & 100 & 40 & 100 & 100 & 0 & \\
\hline & LR (\%) & 20 & 100 & 60 & 100 & 50 & 100 & 40 & 100 & 100 & 0 & \\
\hline
\end{tabular}

Table 6: Comparison between original plan and optimized plan of material 2 in multi-MRP and CRP.

\begin{tabular}{|l|l|l|l|l|l|}
\hline & \multicolumn{4}{|c|}{ LOA } & AWA \\
\hline & Original & Optimized & Improved & Optimized & Improved \\
\hline TC & 195300 & 148300 & $24.066 \%$ & 146800 & $24.833 \%$ \\
\hline
\end{tabular}

Table 7: Comparison of total cost between original plan and optimized plan of 2 materials in multi-MRP and CRP. 


\begin{tabular}{|l|l|l|l|l|l|l|l|l|l|l|}
\hline & \multicolumn{9}{|c|}{ Material 1 } & \multicolumn{5}{c|}{ Material 2 } \\
\hline & & \multicolumn{2}{|c|}{ LOA } & \multicolumn{2}{c|}{ AWA } & \multicolumn{3}{c|}{ LOA } & \multicolumn{2}{c|}{ AWA } \\
\hline & Original & Opt. & Imp. & Opt. & Imp. & Original & Opt. & Imp. & Opt. & Imp. \\
\hline PC & 32600 & 30000 & $7.98 \%$ & 33000 & $1.22 \%$ & 65800 & 6500 & $1.22 \%$ & 67000 & $-1.82 \%$ \\
\hline SC & 13900 & 17700 & $-27.34 \%$ & 16200 & $16.54 \%$ & 5000 & 6600 & $-32 \%$ & 6600 & $-32 \%$ \\
\hline OOSC & 2500 & 0 & $100 \%$ & 0 & $100 \%$ & 29000 & 5000 & $82.76 \%$ & 0 & $100 \%$ \\
\hline PTLO & 6.67 & 0 & $100 \%$ & 0 & $100 \%$ & 3.58 & 1.1 & $69.27 \%$ & 0 & $100 \%$ \\
\hline NTLO & 5.2 & 11 & $111.54 \%$ & 12 & $130.76 \%$ & 3 & 5.6 & $86.67 \%$ & 6.6 & $120 \%$ \\
\hline PTSS & 13.9 & 17.7 & $-27.34 \%$ & 16.2 & $-16.54 \%$ & - & - & - & - & - \\
\hline NTSS & 0 & 0 & $0 \%$ & 0 & $0 \%$ & - & - & - & - & - \\
\hline
\end{tabular}

Table 8: Comparison of all kinds of evaluation of 2 materials in multi-MRP and CRP.

\section{Conclusions}

For the problem that conventional MRP is inconsistent with CRP, MRP and CRP algorithm is designed consistently, configured integrally and used to optimize their resource in this paper, and builds a multi-objective programming model of multi-MRP and CRP based on genetic algorithm. Lexicographic ordering approach [3] and adaptive weight approach [3] are employed to design fitness function; Moreover, $(\mu$ $+\lambda$ ) selection [2] and $N$ points' crossover and mutation are utilized. All above achieve the goals of minimizing the cost of production, stock and OOS, and make the best use of the productive resource. Compared with the conventional MRP algorithm, the integrated optimization of MRP and CRP not only can improve the dependability of CRP but also make total cost decline by $24 \%$, overload decline by $100 \%$ and normal load improved by more than $120 \%$. Integrated optimization algorithm of MRP and CRP, which can be implemented by software and be directly compared with the conventional algorithm, could help managers make decision better.

\section{References}

[1] Z. Chen, L. Yang, Y. Liu and H.Y. Mao, Theory and Application of ERP, Publishing House of Electronics Industry, 2003.

[2] M. Gen and R. Cheng, Genetic Algorithms and Engineering Designment, Publishing Company of Science and Technology, 2000.

[3] M. Gen and R. Cheng, Genetic Algorithms and Engineering Optimization, Tsinghua University Press, 2000.

[4] H.C Jiao, Research of MRP with resource configuration optimization in ERP, Master paper of SCUT, 2006.

[5] H.M. Ye, Enterprise Resource PlanningManufacturing Management, Publishing House of Electronics Industry, 2001.
[6] C.G. Zhou and Y.C. Liang, Computational Intelligence, Jilin University Press, 2005.

[7] Vu T. Le, Bruce M Gunn and S. N. Memeber, IEEE, MRP - Production Planning in Agile Manufacturing. Second IEEE International Conference on Intelligent Systems, 2004. 